\title{
Can Doctors Be App Developers? Our Experience As Medical Educators
}

Goh Kian Liang, Mohd Shahidan Noor Rahin, Ardilla Hanim Abdul Razak, Mohd Shukrimi Awang

International Islamic University Malaysia.

Introduction: Smartphone and mobile apps have become an indispensable companion in our modern-day living and working environment. In the medical field, web browsers and medical apps have transformed the medical practice as the most current knowledge, best practices, medical references, and calculators are available instantaneously at our fingertips. However, these functionality and usage are limited by the creativity and imagination of the app developers. To overcome these limitations, doctors should think out-of-the-box and educate themselves to be appdevelopers to create functional and useful apps in their field. Our aim was to create medical apps from ground-up independently without formal software development training. Material and method: Hybrid app technology is a popular method for creating apps for newbies due to the familiarity with web browsers. We explore the IonicÂA Framework which is a free and open-source front-end with good resources and documentation. This programming technology assumes that everyone has experience with web browsers and the modern browsers essentially run on three technology stacks which are Hypertext Markup Language (HTML), JavaScript and Cascading Style Sheets (CSS). The HTML is a standard markup language for web browser while JavaScript is a scripting language for running commands and functions. The CSS is used for describing how HTML elements should be displayed. Results: We successfully published three apps on the Apple AppStore and Google Play, namely, OrthoBoard $\odot$, OpList and OOLH. Based on our experience of designing, coding, testing and deploying the apps, it is not impossible for medical doctors to achieve this goal and utilizing the app-development skills as an armamentarium for teaching, learning, and medical practice tools. Conclusions: App development is an essential skill especially for medical educators to leverage the technological advancement in their teaching and learning as well as medical practice in tandem with our nations' aspiration towards Education 4.0. 